\title{
Analysis of Unsteady Flow Structures in a Centrifugal Impeller Using Proper Orthogonal Decomposition
}

\author{
Z. Y. Liao ${ }^{1,2,3}$, J. Yang ${ }^{1,2,3 \dagger}$ X. H. Liu ${ }^{4}$, W. L. Hu ${ }^{1}$ and X. R. Deng ${ }^{1}$ \\ ${ }^{1}$ School of Energy and Power Engineering, University of Shanghai for Science and Technology, No. 516 \\ Jungong Road, Shanghai, 200093, China \\ ${ }^{2}$ Key Laboratory of Fluid and Power Machinery of Ministry of Education, Xihua University, No.9999, \\ Hongguang Road, Chengdu 610039, China \\ ${ }^{3}$ Shanghai Key Laboratory of Multiphase Flow and Heat Transfer in Power Engineering, No. 516 Jungong \\ Road, Shanghai, 200093, China \\ ${ }^{4}$ School of Aeronautics and Astronautics, Shanghai Jiao Tong University, No. 500 Donchuan Road, Shanghai \\ 200240, China
}

†Corresponding Author Email: yangjun@ usst.edu.cn

(Received December 30, 2019; accepted July 4, 2020)

\begin{abstract}
The occurrence and development of the dominant unsteady flow structures in a vanless centrifugal pump impeller are revealed by the proper orthogonal decomposition (POD) method. The pressure and velocity data of four radial surfaces is selected as the variables of decomposition. The results show that this method is beneficial to the analysis of flow field when there is no strong interaction of flow structures. When the flow rate starts to decrease from the design flow rate, unstable flow phenomenon such as flow separation and wake begin to appear and develop in the impeller. The POD analysis reveals the influence of the main unsteady structures on the flow field when there is no mixed or have little interaction among flow structures. It outlines the development of flow separation near the suction side of impeller and the wake near the trailing edge as the flow rate changes. However, the flow field inside the impeller becomes more and more complex as the operation condition is far away from the design condition, which needs to be combined with other methods to better analyze the flow field.
\end{abstract}

Keywords: Proper orthogonal decomposition; RANS simulation; Unsteady flow structure; Centrifugal pump impeller.

\section{NOMENCLATURE}

POD eigenvector POD temporal coefficient outlet width of impeller temporal correlation matrix inlet diameter of impeller outlet diameter of impeller time step frequency blade passing frequency rotational frequency head number of snapshots rotating speed a matrix consists of fluctuation part in time order

$Q_{\text {Des }} \quad$ design flow rate

Span non-dimensional distance

St Strouhal number

$S t_{\mathrm{BPF}}$ Strouhal number for the blade passing frequency

\begin{tabular}{|c|c|}
\hline$S t_{\mathrm{R}}$ & Strouhal number for the rotating frequency \\
\hline$S t_{0.23}$ & $\begin{array}{l}\text { a dimensionless frequency its Strouhal } \\
\text { number is } 0.23\end{array}$ \\
\hline$S t_{0.19}$ & $\begin{array}{l}\text { a dimensionless frequency its Strouhal } \\
\text { number is } 0.19\end{array}$ \\
\hline$t_{i}$ & discrete moment \\
\hline$U$ & a matrix consists of snapshots of flow fields \\
\hline $\begin{array}{l}u \\
v\end{array}$ & $\begin{array}{l}\text { at certain discrete moments } \\
\text { outlet circumference velocity of impeller } \\
\text { velocity }\end{array}$ \\
\hline $\bar{v}$ & base flow of flow field \\
\hline$\tilde{v}$ & fluctuation part of flow field \\
\hline$x$ & grid node \\
\hline$Z$ & number of blades \\
\hline$\eta$ & energy ratio \\
\hline$\lambda$ & POD eigenvalue \\
\hline$\varphi$ & POD basis function \\
\hline
\end{tabular}




\section{INTRODUCTION}

Stable and safe operation of centrifugal pumps arise seriously attention as it usually deviates the best efficiency point (BEP) due to engineering needs. The centrifugal impeller is the core component of the centrifugal pump. In such case, the internal flow field of centrifugal impeller is quite complex due to the existence of various unsteady flow phenomena, such as flow separation, recirculation, cavitation and rotational stall (Wang and Tsukamoto 2003, Brun and Kurz 2005, Cheah et al. 2007, X. Li et al. 2018), which is detrimental to the capability and integrity of the centrifugal pump. Therefore, it is an engineering significance to analyze the unsteady flow structures inside centrifugal impeller.

Pressure fluctuations and unsteady flow structures are closely related. Their characteristics can be captured in analysis of quantity of the flow field, such as pressure and velocity. Hence, analyzing the characteristics of pressure fluctuation is a direction to understand the mechanism of the unsteady phenomenon. Both experiments and numerical simulation are capable of the data for subsequent analysis, and the comparison between the results show good agreements (Spence and AmaralTeixeira 2008). In past years, a few researches were carried out numerically or experimentally on the analysis of unsteady flow structures in hydraulic machinery. Pavesi et al. (2008) used auto-spectra and cross-spectra analysis to highlight the frequency and time-frequency domain characteristics of pressure pulsation signals, and the unsteady flow structure and all its characteristics in the centrifugal pump were identified. Li et al. (2016) analyzed the variation of pressure fluctuations in the vaneless region of a pumpturbine based on the validation of the experiments. Cui et al. (2015) demonstrated that the uneven distribution of pressure pulsations around the volute was caused by the rotor/stator interaction between the impeller and the volute. Yang et al. (2013, 2018) studied the rotating characteristics in a pumpturbine under pump mode by spectral analysis, which covered the impeller, diffuser and return channel, and the detail mechanism of rotating stall is revealed. D. Li et al. (2018) confirmed the pressure distribution and propagation characteristics in a pump-turbine for the components of different frequencies through diameter modes theory.

Since the internal flow field becomes complex, traditional analysis based on the raw data is unable to uncover the dominant unsteady flow structures efficiently. The proper orthogonal decomposition (POD) method attracted attentions because of its capability to extract the most energetic unsteady flow structures. After Lumley (1967) successfully applied the POD on the study of turbulence, it remained one of the most widely used techniques and evolved in multiple forms (Sirovich 1987, Christensen et al. 1999, Willcox 2006, Ilak and Rowley 2008, Towne et al. 2018). Liberge and Hamdouni (2010) investigated the circular-cylinder flow a classic fluid mechanics problem, demonstrating that the POD technique could precisely describe its characteristics. POD was also applied to experimental data, i.e. the stereoscopic Particle Image Velocimetry (PIV) (Meyer et al. 2007, Tabib and Joshi 2008, Prothin et al. 2014), as well as the analyses of engine (Fogleman et al. 2004, Semlitsch et al. 2014) and compressor (Semlitsch and Mihăescu 2016) flows. Nevertheless, the application of POD in the analysis of unsteady flow structures inside rotary hydraulic machinery is still in its infancy. Rudolf and Štefan (2012), Stefan and Rudolf (2015), Bistrian and Susan-Resiga (2016) applied POD to investigate the information of swirling flow in the draft cone for different operating conditions. Witte et al. (2018) simulated the flow in a radial turbomachine and applied the POD method to extract the coherent flow structures corresponding to the blade passing frequency. Their work encouraged the analysis of unsteady flow mechanisms in hydraulic turbines from a new perspective.

The POD method is promising for the study of internal flow structures in hydraulic machinery. In this article, the dominant unsteady flow structures inside a centrifugal pump impeller are extracted by the POD methodology. The occurrence and development of those flow structures with operating condition changes are outlined from the perspective of energy and time-space. This research provides a theoretical basis for making a diagnostic analysis using the POD method for the study of the unsteady flow characteristics in rotary hydraulic machinery.

\section{NUMERICAL SETUP}

A classic vanless centrifugal pump with low specific speed is selected, as shown in Fig. 1. The centrifugal pump consists of a closed centrifugal impeller, volute, inlet channel, and outlet channel. The closed impeller has six backward swept blades, of which the design parameters are shown in Table 1. Water is selected as the working fluid during the analysis.

Table 1 Main parameters of the centrifugal

\begin{tabular}{|c|c|}
\multicolumn{2}{|c|}{ impeller } \\
\hline Parameter & value \\
\hline$D_{1} / \mathrm{mm}$ & 79 \\
\hline$D_{2} / \mathrm{mm}$ & 140 \\
\hline$b_{2} / \mathrm{mm}$ & 15.5 \\
\hline$Z$ & 6 \\
\hline$n / \mathrm{r} \cdot \mathrm{min}^{-1}$ & 2910 \\
\hline$Q_{\text {Des }} / \mathrm{m}^{3} \cdot \mathrm{h}^{-1}$ & 50.6 \\
\hline
\end{tabular}

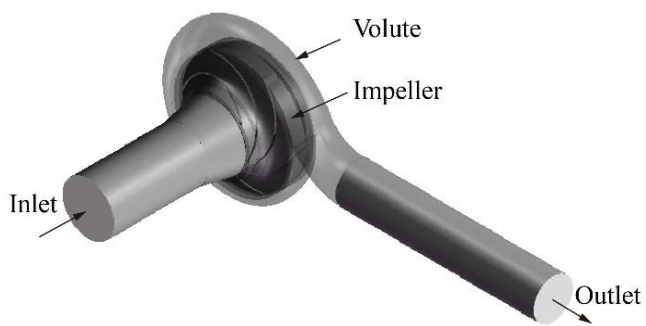

Fig. 1. Geometry model of the vanless centrifugal pump. 
Several hexahedral meshes are used in the simulation, their total number of grids are around 1.3 million, 0.8 million, 2.3 million, 2.8 million and 3.3 million, respectively. In order to verify the accuracy of calculation and the influence of grid on calculation results, the head is chosen to make a comparison between experimental and numerical simulation results under design flow rate, which is one of the main performance parameters in centrifugal pump. The details are shown in Fig. 2. Obviously, with the increase of the number of grids, the calculated value of head becomes steady and closer to the experimental value. Considering the precision of numerical calculation and the consumption of computing resources, the hexahedral mesh with a total cell number of $2.8 \times 10^{6}$ is used for analysis in this paper. The number of grids for each part are listed in table 2 .

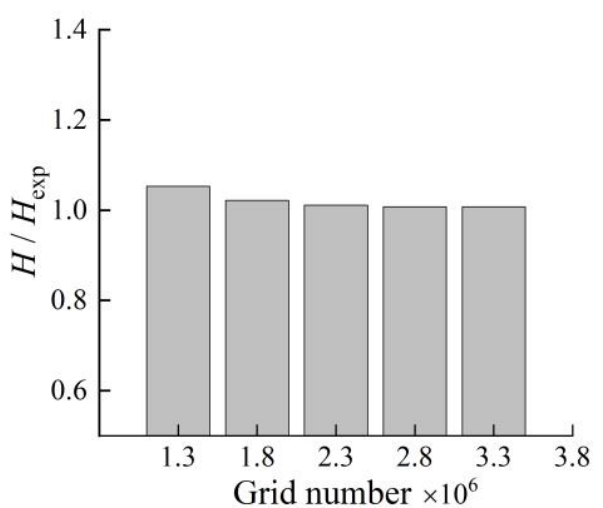

Fig. 2. Grid independence validation under design flow rate.

Table 2 Number of grids for each part

\begin{tabular}{|c|c|}
\hline Part & Grid number $/ \times 10^{6}$ \\
\hline Impeller & 1.06 \\
\hline Volute & 0.88 \\
\hline Inlet channel & 0.44 \\
\hline Outlet channel & 0.42 \\
\hline
\end{tabular}

The numerical simulations are carried out for several operating conditions (1.0 $Q_{\text {Des, }} 0.8 Q_{\text {Des, }} 0.7$ $Q_{\text {Des }}$ and $0.6 Q_{\text {Des }}$ ), performed by a commercial code ANSYS CFX. In this research, the finite volume method with High resolution scheme is selected, and the SST k- $\omega$ turbulence model is used. The interface between the rotating domain and the stationary domain is set as Transient Rotor Stator. The time for impeller rotates three degrees $(0.0001718 \mathrm{~s})$ is chosen as the time step size. The mass flow rate is specified at the inlet according to the experimental data, and the opening boundary is chosen at the outlet. To achieve a better convergence, the results of steady state simulation are used as the initial conditions for the unsteady calculation.

In order to investigate the characteristics of the unsteady flow structures in more detail, four radial surfaces are sequentially established along the direction from hub to shroud for sampling data, as shown in Fig. 3.
The radial surfaces on impeller are described by a non-dimensional distance Span value, defined as the ratio of the distance between the radial surface and hub and the distance between hub and shroud. Hence, Span $=0$ indicate hub and Span $=1$ indicate shroud. The four radial surfaces are named as Span 0.1, Span 0.5, Span 0.7, and Span 0.95.
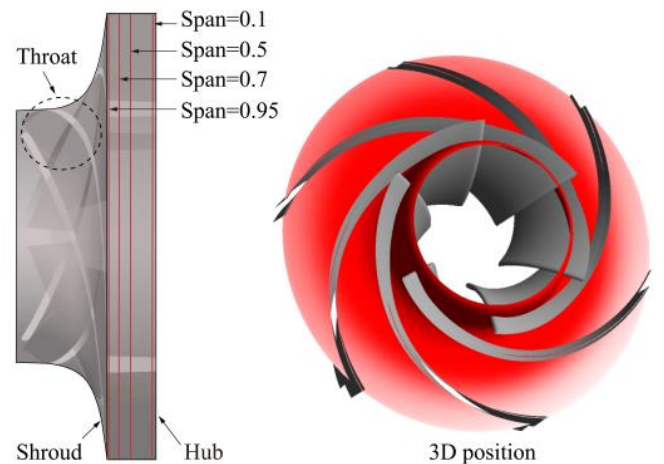

Fig. 3. Radial surfaces in the impeller and its shape.

In the research, all frequencies are described as a function of Strouhal number $(S t)$ which is a nondimensional and based on the circumferential distance between two blades and the impeller tip speed in the circumferential direction (Yang et al. 2018), calculated via Eq. (1).

$$
S t=\frac{\pi D_{2}}{Z} \frac{f}{u}=\frac{f}{f_{\mathrm{BPF}}}
$$

Where $D_{2}$ is the outlet diameter of the impeller, the unit is $\mathrm{m} ; Z$ is the number of blades; $u$ is the outlet circumference velocity of impeller, the unit is $\mathrm{m} \cdot \mathrm{s}^{-1}$; $f$ is the frequency, the unit is $\mathrm{Hz} ; f_{\mathrm{BPF}}$ is the blade passing frequency, the unit is $\mathrm{Hz}$.

Specifically, the blade passing frequency $f_{\mathrm{BPF}}$ and the rotating frequency $f_{R}$ of this pump is $291 \mathrm{~Hz}$ and $48.5 \mathrm{~Hz}$ respectively, that is $S t_{\mathrm{BPF}}=1$ and $S t_{\mathrm{R}} \approx$ 0.1667 . Moreover, the sampling frequency is 5820 $\mathrm{Hz}$ (one of the time step) is much larger than 10 times of the dominant frequency $f_{\mathrm{BPF}}$ in system, hence the Nyquis Sampling Theorem is fulfilled (Yuan et al. 2012).

\section{RESEARCH METHODS}

In this research, the Fourier transform method is used to analyze the one-dimensional flow characteristics in the frequency domain to obtain a preliminary conclusion about the unsteady flow characteristics in the flow field. Then the proper orthogonal decomposition (POD) based on the snapshots of feature quantities (velocity and pressure) in the flow field is carried out to extract the high-energy flow modes in order to further analyze the flow structure characteristics in the flow field. The specific implementation process is described below.

The matrix composed of snapshots of characteristic quantity of flow field at a set of discrete moments 


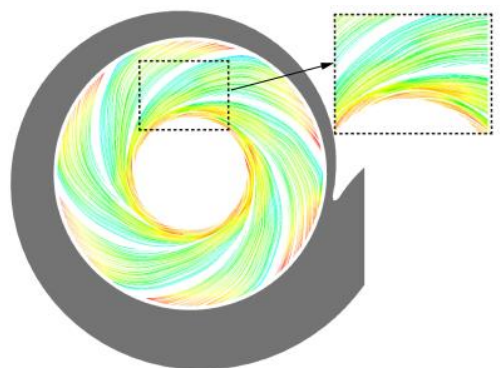

(a)



(c)

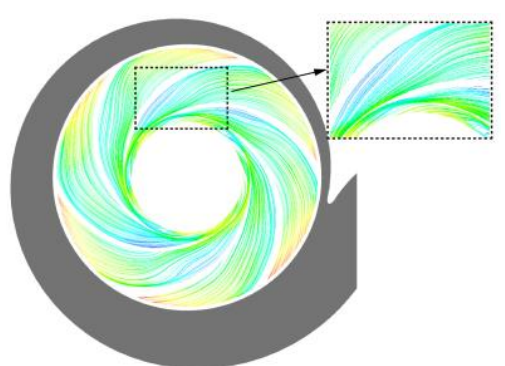

(b)

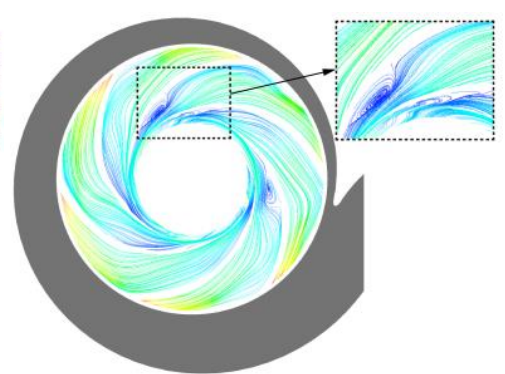

(d)

Fig. 4. 2-D flow distribution of impeller: (a) $1.0 Q_{\text {Des, }}$, (b) $0.8 Q_{\text {Des, }}$ (c) $0.7 Q_{\text {Des, }}$ and (d) $0.6 Q_{\text {Des. }}$

on monitoring face (such as the radial surfaces display in Fig. 3) is obtained through simulation. In this section, velocity is selected as the flow field characteristic to taken as an example. The matrix $U$ is shown as Eq. (2).

$$
U=\left\{v\left(x, t_{i}\right)\right\}_{i=1}^{m}
$$

where $v$ is the velocity; $x$ is the grid node; $t_{i}$ is the discrete moment; $m$ is the number of snapshots.

The flow field at any moment can be expressed as a juxtaposition of a base flow part $\bar{v}\left(x, t_{i}\right)$ and a fluctuation part $\tilde{v}\left(x, t_{i}\right)$, as shown in Eq. (3).

$$
v\left(x, t_{i}\right)=\bar{v}\left(x, t_{i}\right)+\tilde{v}\left(x, t_{i}\right)
$$

The POD technique makes the fluctuation part decomposed into an orthonormal system of POD basis function $\varphi_{j}$ and POD temporal coefficient $a_{j}$, as shown in Eq. (4).

$$
\tilde{v}\left(x, t_{i}\right)=\sum_{j=1}^{m} a_{j}\left(t_{i}\right) \cdot \varphi_{j}(x)
$$

where $a_{j}$ is a row vector arranged in time series, reflecting the temporal characteristic of the $j$ th POD mode; $\varphi_{j}$ is a column vector corresponding to the grid nodes, reflecting the spatial distribution of the $j$ th POD mode.

Equations (5), (6) and (7) describe the core of the POD, which is the eigenvalue decomposition of the temporal correlation matrix $C$.

$$
\begin{aligned}
C & =P^{T} P \\
P & =\left[\tilde{v}\left(x, t_{1}\right), \tilde{v}\left(x, t_{2}\right), \ldots, \tilde{v}\left(x, t_{m}\right)\right]
\end{aligned}
$$

$C A_{j}=\lambda_{j} A_{j}, j=1,2 \ldots m$

where matrix $P$ consists of the fluctuation part in time order; $\lambda_{j}$ and $A_{j}$ are the POD eigenvalues and its corresponding eigenvector respectively. The POD eigenvalue calculated from Eq. (7) signifies the perturbation energy of the POD mode. According to the ranking of POD eigenvalues, the energy distribution of each POD mode could be drawn in order from high to low, as shown in Fig. 6 and Fig. 7. The energy contribution of each POD mode to the target flow field can be obtained from these figures.

Each POD mode has a basis function $\varphi_{j}$ and a temporal coefficient $a_{j}$, which are calculated by Eq. (8) and (9), respectively.

$$
\begin{aligned}
& \varphi_{j}=P A_{j} / \sqrt{\lambda_{j}} \\
& a_{j}=\varphi_{j}^{T} P
\end{aligned}
$$

In this step, for every monitoring surface, the temporal coefficient $a_{j}$ of each energetic POD mode is obtained by Eq. (9). Then Fourier transform is applied to this temporal coefficient, and the detail results are illustrated as Fig. 8 and Fig. 11. According to these graphs, the main discrete frequency of the corresponding flow field on this mode can be obtained. At the same time, these results also are compared with the frequency domain results of flow characteristics of flow field monitoring points (the results are shown in Fig. 5) to verify the feasibility and reliability of this method.

According to the above frequency analysis, the basis function $\varphi_{\mathrm{j}}$ of the energetic POD mode with 


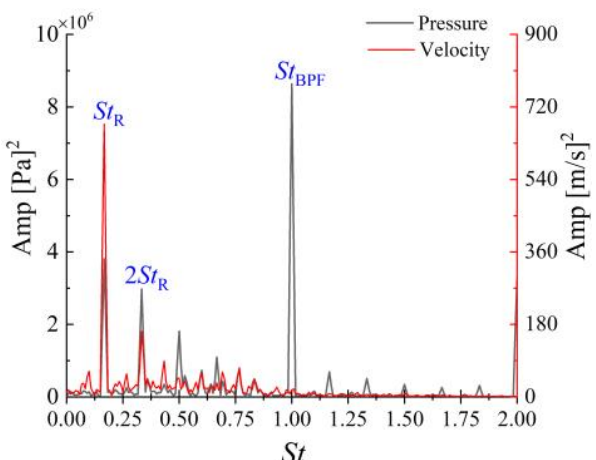

(a)



(b)

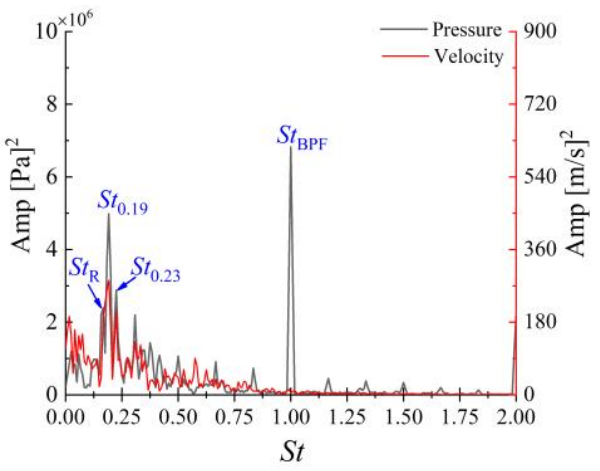

(c)

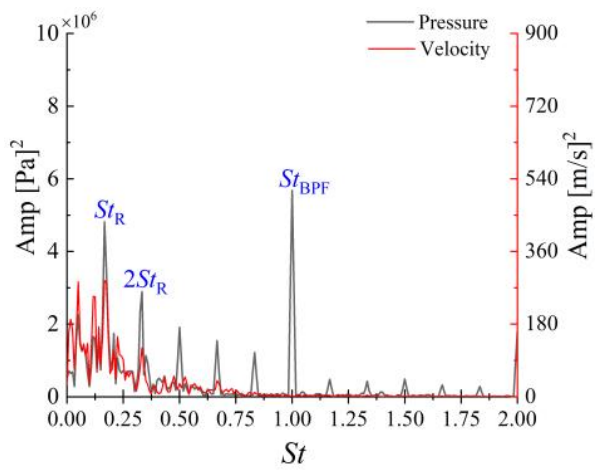

(d)

Fig. 5. Spectrum of the velocity and pressure in the blade passage: (a) $1.0 Q_{\text {Des, }}$ (b) $0.8 Q_{\text {Des, }},(c) 0.7 Q_{\text {Des, }}$ and (d) $0.6 Q_{\text {Des. }}$

dominant frequencies, obtained according to Eq. (9), is visualized in conjunction with grid node coordinates, thereby obtaining the high amplitude distribution of the flow mode, as shown in Fig. 9, Fig. 10, and Fig. 12 to Fig. 14.

\section{ReSults AND Discussion}

\subsection{Flow Field Distribution Inside the Centrifugal Impeller}

The Two-dimensional flow distribution of impeller under different flow rates (1.0 $Q_{\text {Des, }}, 0.8 Q_{\text {Des, }}, 0.7$ $Q_{\text {Des }}$ and 0.6 $\left.Q_{\text {Des }}\right)$ are shown in Fig. 4. The flow field distribution is relatively even under 1.0 $Q_{\text {Des. }}$ As the flow rate decreases, the flow field distribution in the throat becomes inhomogeneous. At the same time, the flow separation phenomenon intensifies with the further reduction of the flow rate, resulting in further deterioration of the flow inside the impeller. In addition, the inlet flow field begins to be uneven, as shown in the flow field

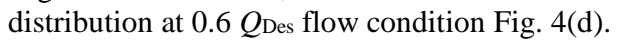

The Fast Fourier transform (FFT) is applied to the velocity and pressure signals sampled in some monitoring points being in the blade passage. One of the results is shown in the Fig. 5. The main discrete peaks appeared at $S t_{\mathrm{BPF}}, S t_{\mathrm{R}}$ and their higher harmonics for the operating condition 1.0 $Q$ Des. When the flow rate decreases, the perturbations at low-frequencies are amplified $(S t \leq$
$0.5)$, which may be related to the occurrence of flow separation.

However, so many low-frequency discrete peaks are not easy to analyze in these pictures. Therefore, POD analysis has been carried on the flow field data of the numerical simulation results. By analyzing these modes which account for the main proportion of the energy in the next section, the flow structures which play leading roles in the impeller under these conditions are analyzed in the subsequent sections.

\subsection{Energy Distribution}

The perturbation energy distribution of POD modes is reflected by energy ratio $\eta$ calculated via Eq. (10).

$$
\eta=100 \times \lambda_{j} / \sum_{1}^{m} \lambda_{i}, j=1,2, \ldots, m
$$

where $\lambda_{\mathrm{j}}$ is the POD eigenvalue. The perturbation energy distribution of POD modes of pressure and velocity field are shown in Fig. 6 and Fig. 7.

In these pictures, after ranking POD modes based on its energy ratio, for each radial section, the total energy ratio of first 20 modes has been listed and the first mode has the largest energy.

However, it can be seen from Fig. 7 that even the 20 order POD modes of velocity field together do not account for $60 \%$ of the energy of the whole 


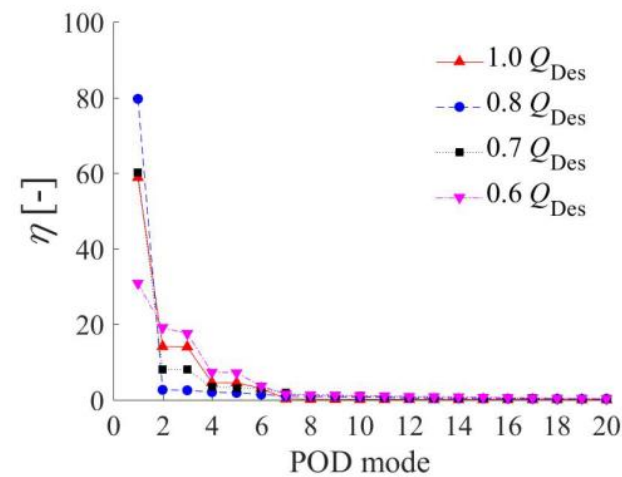

(a)



(c)

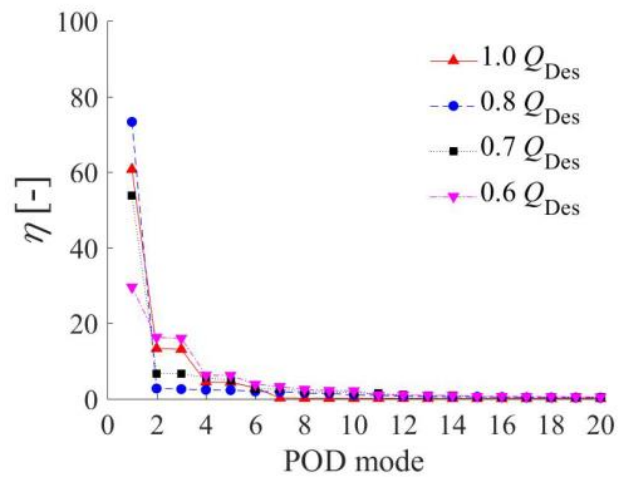

(b)



(d)

Fig. 6. Energy distribution of POD modes of pressure field of Span 0.1 (a), Span 0.5 (b), Span 0.7 (c), and Span $0.95(d)$.

monitoring surface. Therefore, it is not sufficiently credible to determine the flow structure that plays a dominant role in this surface through the analysis of the results of several velocity modes obtain by POD method.

In contrast, the perturbation energy distribution of the modes of pressure field in Fig. 6 is relatively concentrated, the energy of the first five modes under the above four operating conditions accounts for nearly $90 \%$ of the total perturbation energy. Therefore, we intend to analyze which flow structure plays the main role in the flow field as the flow rate decreases by using pressure data for POD first, then the POD results of velocity field are analyzed to get more specific flow details.

\subsection{POD Analysis of Pressure Field}

In order to explore the characteristics of the main unsteady flow structure at partial flow rates, the first two pressure POD modes of each monitoring surface are selected for the following analysis due to its high energy ratio. The corresponding time coefficients of the $1^{\text {st }}$ and $2^{\text {nd }}$ POD mode of pressure field are analyzed through FFT method to obtain the frequency characteristics of these modes, as shown in Fig. 8. There are multiple discrete peaks in these spectrograms, indicating that there are multiple flow structures in a same mode because it is ordered by energy contribution level.
Obviously, it can be inferred from Fig. 8 that the blade passing frequency $S t_{\mathrm{BPF}}$ caused by the rotor stator interaction (RSI) is the dominant frequency of the $1^{\text {st }}$ pressure mode of every surface. The rotating frequency $S t_{\mathrm{R}}$ is the dominant frequency of $2^{\text {nd }}$ mode under design flow condition. However, when the flow condition reduces to $0.8 Q_{\text {Des }}$ and $0.7 Q_{\text {Des, }}$ as seen in Fig. 8(b) and Fig. 8(c), the perturbation of frequency $S t_{\mathrm{R}}$ gradually weaken and high disturbances appear successively at frequencies $S t_{0.23}(S t \approx 0.23)$ and $S t_{0.19}(S t \approx 0.19)$. When the flow rate is further reduced to $0.6 Q_{\text {Des, }}$, the pulsation of the $S t_{\mathrm{R}}$ is strengthened again while the amplitude at $S t_{0.23}$ and $S t_{0.19}$ are weakened. The spectrum characteristics of these modes are highly agreement with the FFT results of the pressure signal in Fig. 5, which all prove the appearance of the frequency $S t_{0.23}$ and $S t_{0.19}$. The results under 0.8 $Q_{\text {Des }}$ and $0.7 Q_{\text {Des }}$ are focused in this section.

The spatial distribution of the $1^{\text {st }}$ and $2^{\text {nd }}$ POD mode of pressure field of four surfaces at $0.8 Q_{\text {Des }}$ and 0.7 $Q_{\text {Des }}$ are shown in Fig. 9 and Fig. 10. The $1^{\text {st }}$ mode of each surface has the dominant frequency $S t_{\mathrm{BPF}}$, as seen in Fig. 9(a) and Fig. 10(a), and spreads all over the whole blade passage, presenting the main pulsation of the pressure field. For the $2^{\text {nd }}$ mode, obvious local high-amplitude regions appear where may be related to the effect of several unsteady flow structures with different discrete frequency in the corresponding spectrum. The pictures of Fig. 9(b) 
Z. Y. Liao et al. / JAFM, Vol. 14, No. 1, pp. 89-101, 2021.

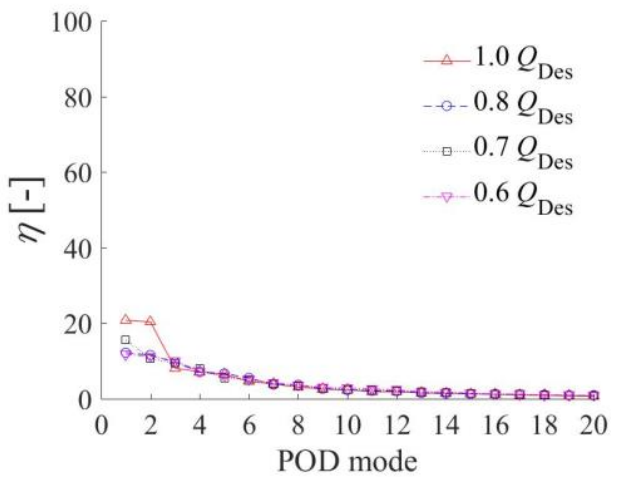

(a)

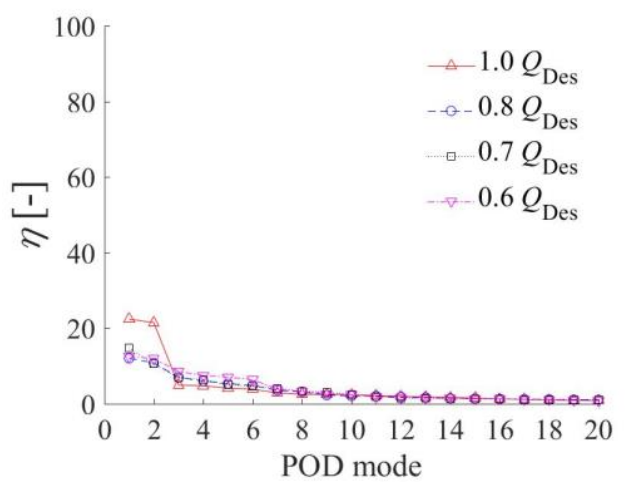

(c)



(b)

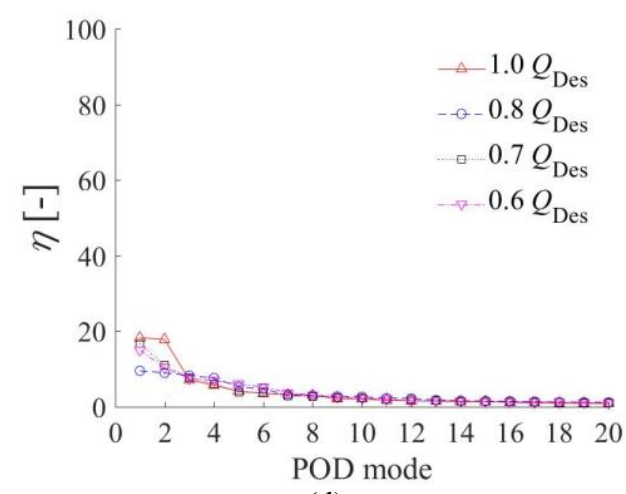

(d)

Fig. 7. Energy distribution of POD modes of velocity field of Span 0.1 (a), Span 0.5 (b), Span 0.7 (c), and Span 0.95 (d).

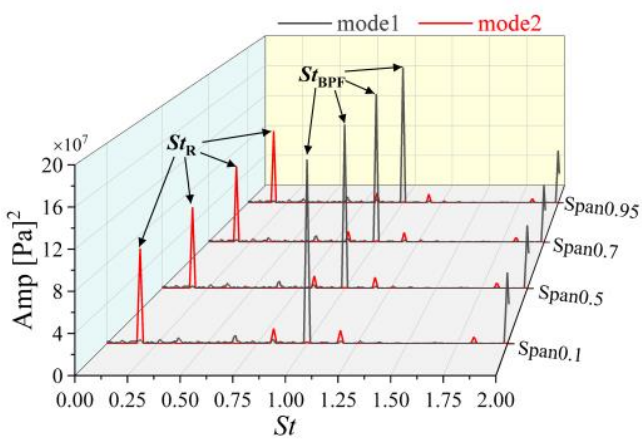

(a)

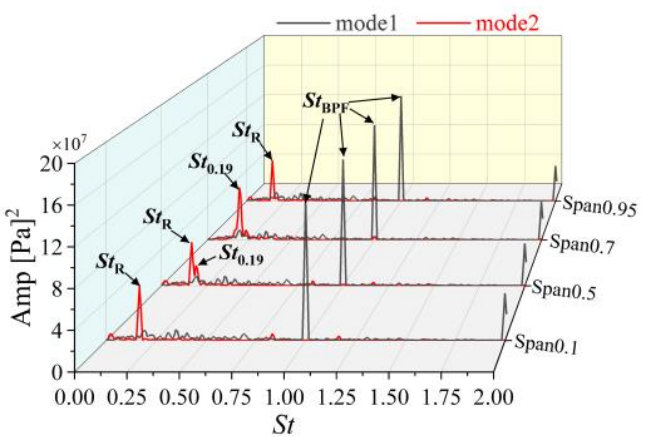

(c)

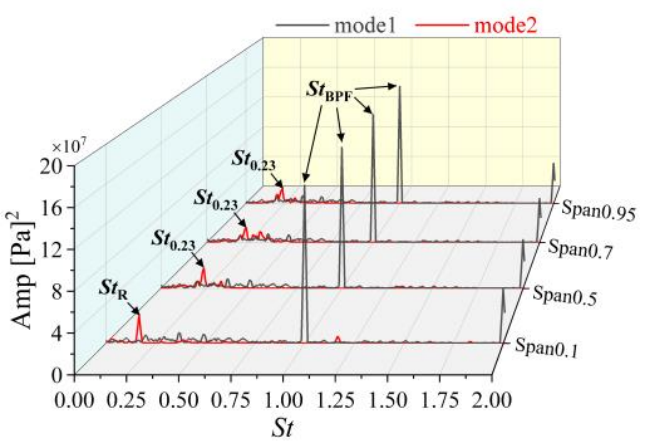

(b)

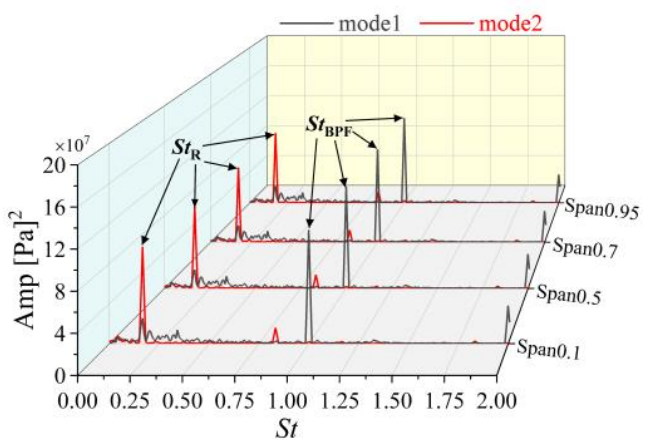

(d)

Fig. 8. Spectrum of the $1^{\text {st }}$ and $2^{\text {nd }}$ POD modes of pressure field under $1.0 Q_{\text {Des }}(\mathrm{a}), 0.8 Q_{\text {Des }}(\mathrm{b}), 0.7 Q_{\text {Des }}$ (c) and 0.6 $Q_{\text {Des }}(d)$. 


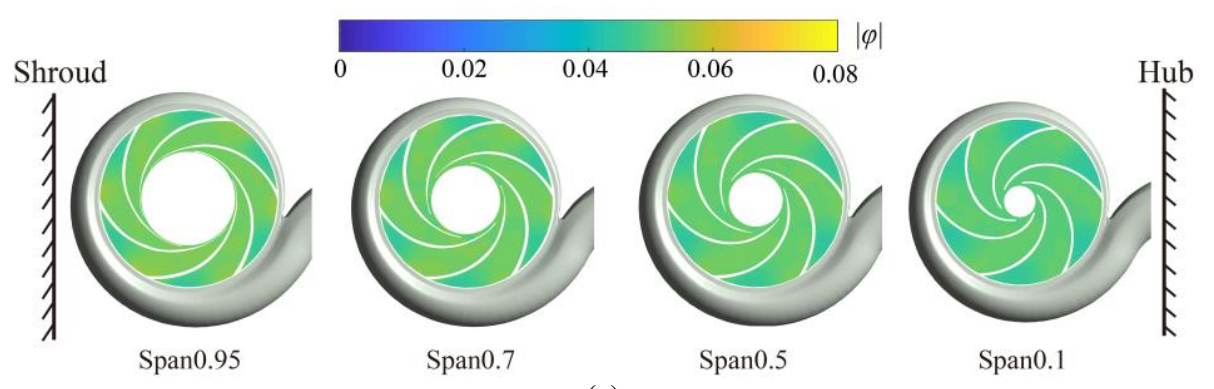

(a)
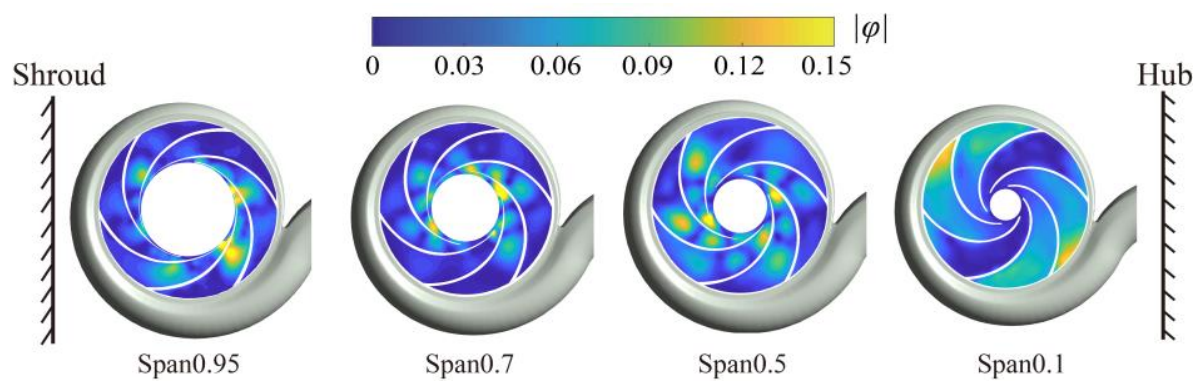

(b)

Fig. 9. Isosurface of the $1^{\text {st }}$ POD mode (a) and the $2^{\text {nd }}$ POD mode (b) of pressure field under $0.8 Q_{\text {Des. }}$

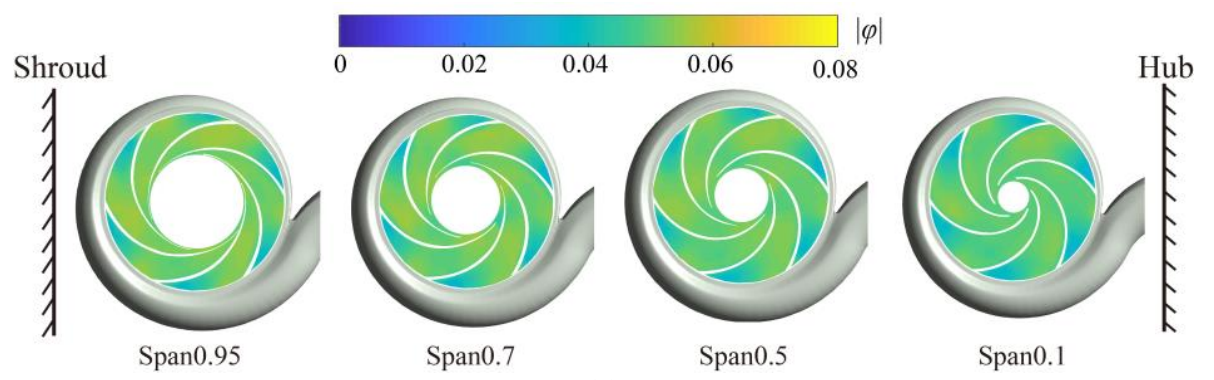

(a)
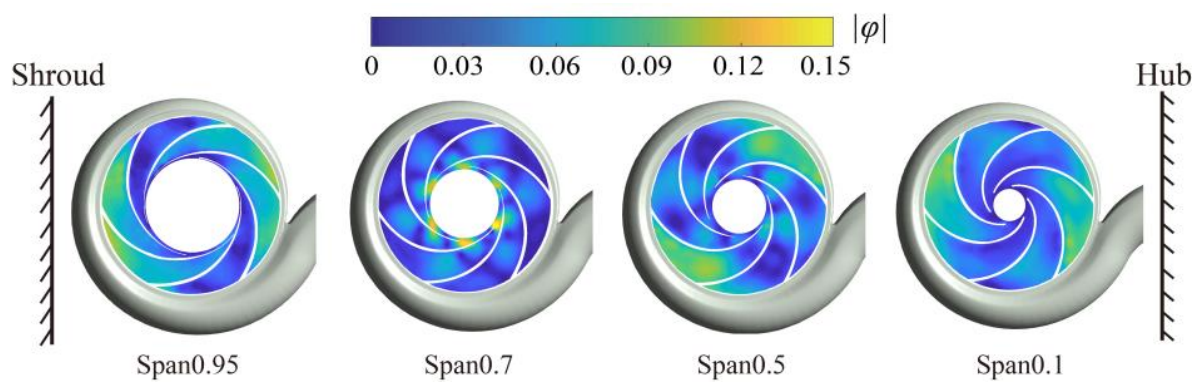

Fig. 10. Isosurface of the $1^{\text {st }}$ POD mode (a) and the $2^{\text {nd }}$ POD mode (b) of pressure field under $0.7 Q_{\text {Des. }}$

shows that under $0.8 Q_{\text {Des }}$ flow condition, the highamplitude regions of the $2^{\text {nd }}$ mode of surfaces Span 0.95 , Span 0.7 and Span 0.5 are mainly distributed in the throat and develop towards the outlet, and there are many unforced flow structures with different discrete frequency but the main one is Sto.23. The frequency $S t_{0.23}$ has been proved to be a nonlinear frequency in the research of Yang et al. (2019) by Bispectrum analysis.
Under $0.7 Q_{\text {Des }}$ flow condition shown in Fig. 10 (b), only the distribution of the $2^{\text {nd }}$ mode of surface Span 0.7 has the same characteristics with the one under $0.8 Q$ Des flow condition. The distribution on surface Span 0.5 also shows similar characteristics but it is not obvious. From Fig. 8(c), the dominant frequency of the $2^{\text {nd }}$ mode of surface Span 0.7 is $S t_{0.19}$, which also has a weak peak in the $2^{\text {nd }}$ mode of Span 0.5 whose dominant frequency is $S t_{R}$, therefore further explaining that the high-amplitude 


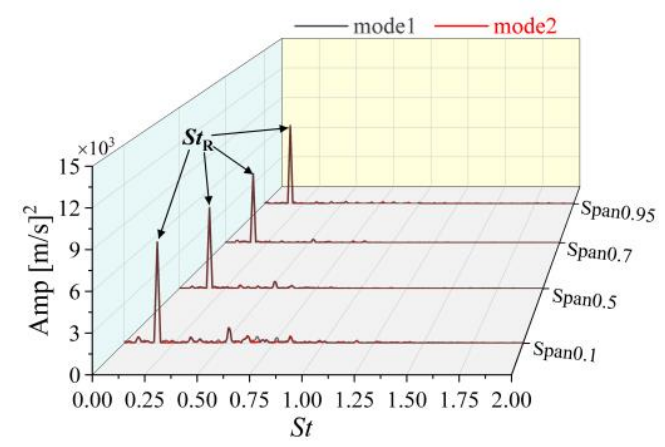

(a)



(c)

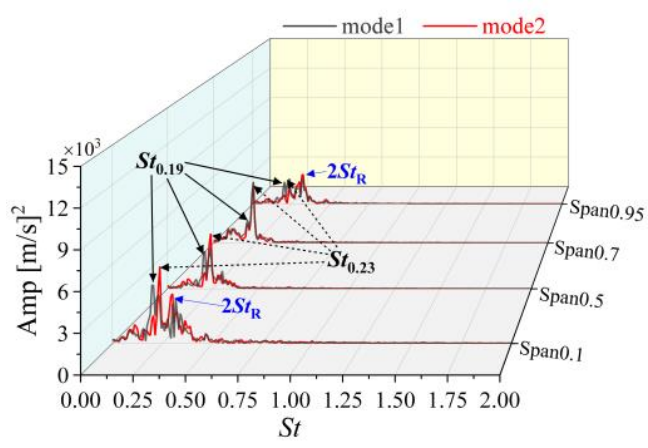

(b)



(d)

Fig. 11. Spectrum of the $1^{\text {st }}$ and $2^{\text {nd }}$ POD modes of velocity field under $1.0 Q_{\text {Des }}(a), 0.8 Q_{\text {Des }}(b), 0.7 Q_{\text {Des }}$ (c) and 0.6 $Q_{\text {Des }}(\mathrm{d})$.

distribution is affected by a combination of multiple flow structures.

According to the above POD analysis of pressure field, it can be summarized that, the $1^{\text {st }}$ mode of each surface is the main provider of the pulsating energy in impeller pressure field under the flow conditions of $0.8 Q_{\text {Des }}$ and $0.7 Q_{\text {Des, }}$ and its distribution spreads all over the impeller channel. For those $2^{\text {nd }}$ POD modes with the dominant frequency $S t_{0.23}$ or $S t_{0.19}$ in the spectrums, its highamplitude region concentrates in the throat and develop towards impeller outlet.

Although the results of pressure field POD analysis initially indicate that unforced flow structures appeared in $0.8 Q_{\text {Des }}$ and $0.7 Q_{\text {Des }}$ and with high perturbation energy, it failed to provide more information about it. Therefore, the POD method is applied to the velocity field for obtaining more details.

\subsection{POD Analysis of Velocity Field}

For comparison and discussion, we also choose the first two order POD modes in velocity field with the highest energy of the surface for analysis. Similarly, we show the frequency spectrum results of the time coefficients of first two modes in Fig. 11, from where the spectral characteristics also are highly agreement with the FFT results of the velocity signals in Fig. 5.

Compared with the results of pressure POD analysis, it more clearly reflects the change of spectrum characteristics as the flow rate decreases, that is, the disturbance of $S t_{\mathrm{R}}$ is weakened while the disturbances of the frequencies $S t_{0.23}$ and $S t_{0.19}$ appear. However, the frequency $S t_{0.19}$ firstly appears under $0.8 Q_{\text {Des }}$ flow condition dominated by the frequency $S t_{0.23}$, and its perturbation is greatly enhanced at $0.7 Q_{\text {Des }}$ where the perturbation of frequency $S t_{0.23}$ still exists but has been weakened. Furthermore, there are new obvious peak frequencies $S t_{0.09}(S t \approx 0.09)$ and $S t_{0.12}(S t \approx 0.12)$ at $0.6 Q_{\text {Des. }}$ These changes cannot be captured by the POD analysis of pressure field.

According to the flow field distribution in Fig. 4, there is a flow separation phenomenon in the throat region of impeller in the mainstream area not close to shroud and hub. Combining with the distribution of the $1^{\text {st }}$ and $2^{\text {nd }}$ POD modes of velocity field under these flow conditions (in Fig. 12 and Fig. 13), we speculate that the frequency $S_{0.23}$ and $S t_{0.19}$ are likely to be the leading characteristic frequencies of this flow separation. It can be clearly found from Fig. 12 that the distribution characteristics of the $1^{\text {st }}$ and $2^{\text {nd }}$ modes under $0.8 Q_{\text {Des }}$ are similar, there are several high-amplitude fluid masses near the suction surface of the blade, corresponding to flow separation phenomenon in Fig. 15. They appear near the entrance region of the impeller of surface Span 0.95, and move towards the impeller outlet in the direction from shroud to hub. Furthermore, there are some high amplitude region near the exit in 

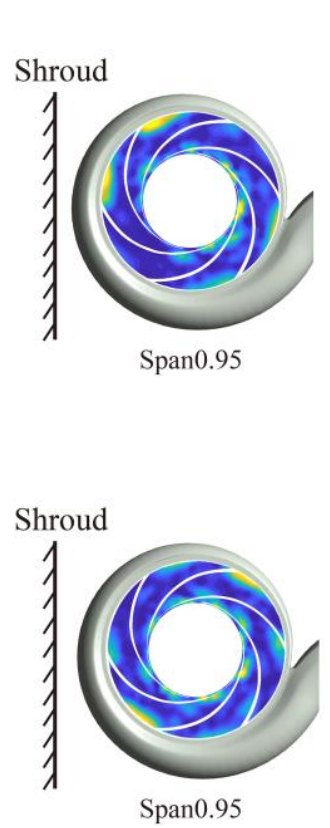


(a)
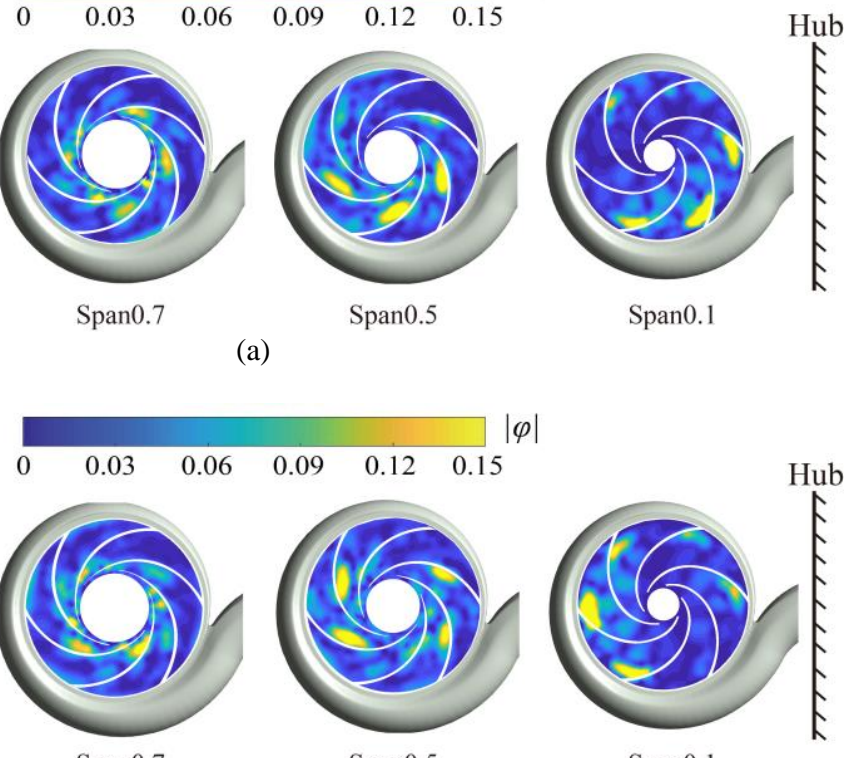

(b)

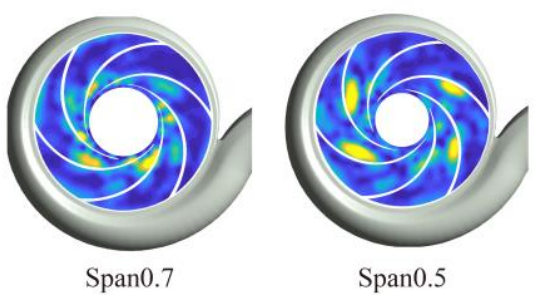

Span0.1

Fig. 12. Isosurface of the $1^{\text {st }}$ POD mode (a) and the $2^{\text {nd }}$ POD mode (b) of velocity field under $0.8 Q_{\text {Des. }}$
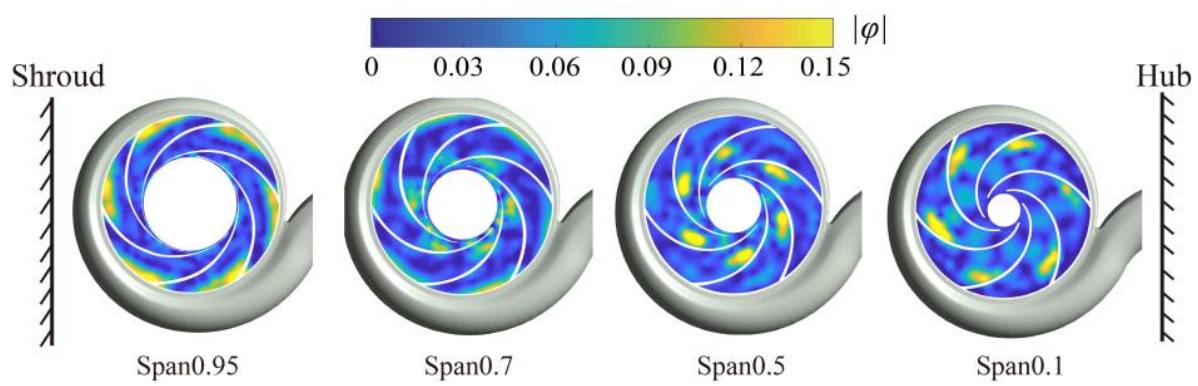

(a)


(b)

Fig. 13. Isosurface of the $1^{\text {st }}$ POD mode (a) and the $2^{\text {nd }}$ POD mode (b) of velocity field under $0.7 Q_{\text {Des. }}$

surface Span 0.95, which is consistent with the wake area compared with the flow distribution in the impeller (Fig. 15), but the jet area is not obvious.

The spatial distribution characteristics of the modes under $0.7 Q_{\text {Des }}$ flow condition do not change much compared with $0.8 Q_{\text {Des. }}$ However, the dominant frequency is changed from $S t_{0.23}$ to $S t_{0.19}$. The quantity of the high-amplitude flow masses near the suction surface of blade increases, as observed in Fig. 13, and the high-amplitude region at the outlet of the surface Span 0.95 is enhanced. At the same time, the jet area is still not obvious. It shows that the flow separation of the blade suction surface and the wake of the impeller outlet are intensified.

As the flow rate further reduces to 0.6 $Q_{\text {Des, }}$ the disturbances of the frequencies $S t_{0.23}$ and $S t_{0.19}$ are reduced. From Fig. 14, there is no distribution characteristic like the other two working conditions. However, there are still higher amplitude distributions in the inlet and throat of impeller and the outlet region of surface Span 0.95. 


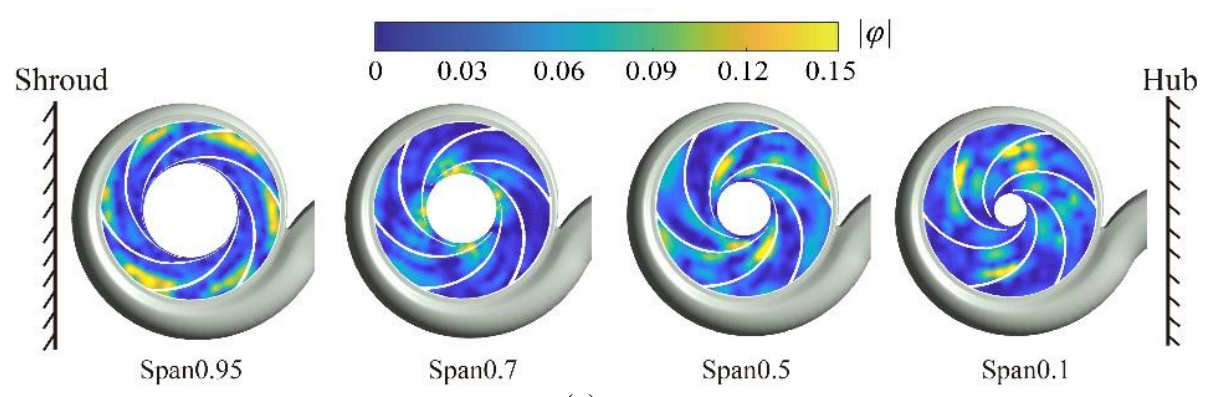

(a)

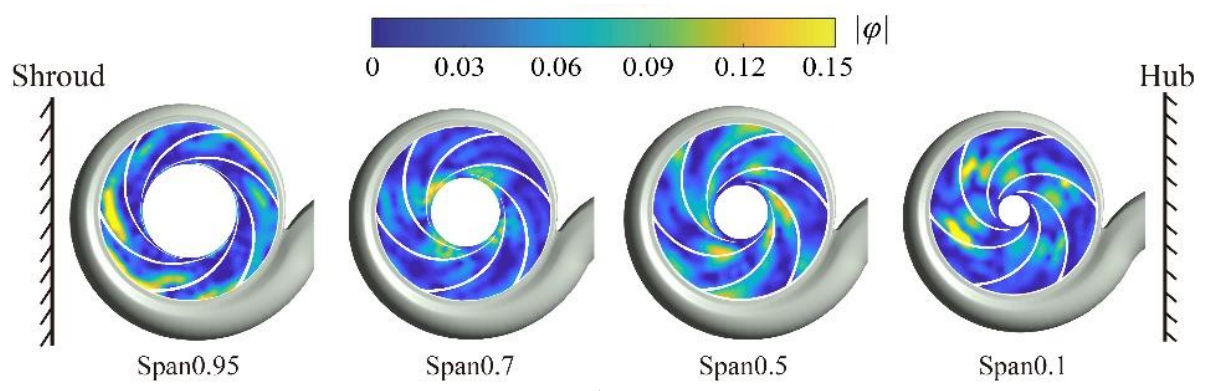

(b)

Fig. 14. Isosurface of the $1^{\text {st }} P O D$ mode (a) and the $2^{\text {nd }} P O D$ mode (b) of velocity field under $0.6 Q_{\text {Des. }}$

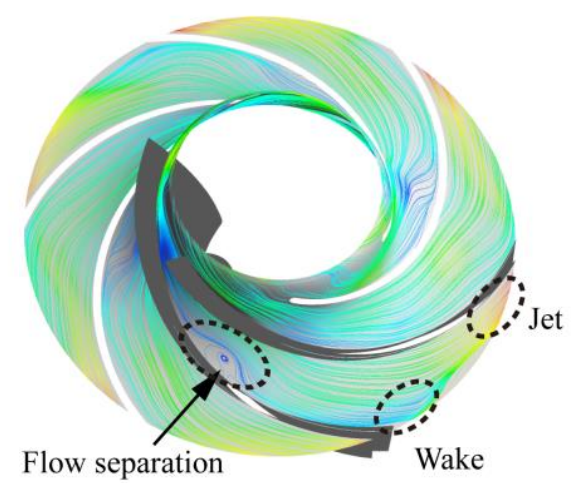

Fig. 15. Phenomenon of flow separation, wake and jet in the impeller.

\section{CONCLUSIONS}

The proper orthogonal decomposition (POD) technique is applied to reveal the energetic flow modes with dominant unsteady flow patterns in a vanless centrifugal pump impeller. The POD analyses were performed for four radial impeller sections at constant span, using pressure and velocity as the decomposition variables for various operating conditions.

As the flow rate decreases from the design flow rate, various unstable flow structures begin to appear inside the impeller, such as flow separation on the suction surface of the impeller and jets and wakes on the trailing edge. According to the POD analyses of pressure field and velocity field, several conclusions about the development of the main unsteady flow phenomena are summarized as follow:
1. As the flow rate began to decrease, the flow separation first appears on the suction surface of the impeller. This flow structure is initially dominated by the disturbance of the frequency $S t_{0.23}$. Then with the flow rate reduces, the frequency $S t_{0.19}$ is developed and its disturbance is highly enhanced. However, the internal flow field becomes more complex as the flow rate further reduces, the frequency $S t_{0.23}$ and $S t_{0.19}$ disappear at $0.6 Q_{\text {Des }}$ condition due to the intense interaction among various unsteady flow structures.

2. The wake phenomenon of the impeller outlet is highlighted near the shroud and gradually intensifying with the flow rate decreases in the results of the POD analysis. However, there is no corresponding high-amplitude region for the jet phenomenon of the impeller outlet that occurred in the flow field distribution. This shows that the influence of wake on the 
impeller flow field is more obvious.

The shortcomings of the POD method are also concluded. This method is advantageous for analyzing those flow field with relatively single flow structure. However, as the flow in the flow field becomes complicated, it is difficult to obtain detailed flow structure information, such as the internal flow of the impeller under the 0.6 $Q_{\text {Des }}$ in this article. Therefore, more different analysis methods need to be contacted to analyze the occurrence and development rules of each flow structure for complex flow fields.

\section{ACKNOWLEDGEMENTS}

This work is supported by the National Natural Science Foundation of China (Grant No. 51976125 and 51976116), the Open Research Subject of Key Laboratory (Fluid Machinery and Engineering Research Base) of Sichuan Province (grant number zj2015-024), and Natural Science Fund of Shanghai (grant No. 19ZR1425900).

\section{REFERENCES}

Bistrian, D. A. and R. F. Susan-Resiga (2016). Weighted proper orthogonal decomposition of the swirling flow exiting the hydraulic turbine runner. Applied Mathematical Modelling 40 (5), 4057-4078.

Brun, K. and R. Kurz (2005). Analysis of Secondary Flows in Centrifugal Impellers. International Journal of Rotating Machinery 2005 (1), 45-52.

Cheah, K. W., T. S. Lee, S. H. Winoto and Z. M. Zhao (2007). Numerical Flow Simulation in a Centrifugal Pump at Design and Off-Design Conditions. International Journal of Rotating Machinery, 83641.

Christensen, E. A., M. Brøns and J. N. Sørensen (1999). Evaluation of Proper Orthogonal Decomposition--Based Decomposition Techniques Applied to Parameter-Dependent Nonturbulent Flows. SIAM Journal on Scientific Computing 21 (4), 1419-1434.

Cui, B., D. Chen, W. Xu, Y. Jin and Z. Zhu (2015). Unsteady flow characteristic of low-specificspeed centrifugal pump under different flowrate conditions. Journal of Thermal Science 24 (1), 17-23.

Fogleman, M., J. Lumley, D. Rempfer and D. Haworth (2004). Application of the proper orthogonal decomposition to datasets of internal combustion engine flows. Journal of Turbulence 5, N23.

Ilak, M. and C. W. Rowley (2008). Modeling of transitional channel flow using balanced proper orthogonal decomposition. Physics of Fluids 20 (3), 034103.

Li, D. Y., R. Z. Gong, H. J. Wang, X. Z. Wei, Z. S. Liu and D. Q. Qin (2016). Analysis of Rotor-
Stator Interaction in Turbine Mode of a PumpTurbine Model. Journal of Applied Fluid Mechanics 9(5), 2559-2568.

Li, D., H. Wang, Y. Qin, Z. Li, X. Wei and D. Qin (2018). Mechanism of high amplitude low frequency fluctuations in a pump-turbine in pump mode. Renewable energy 126, 668-680.

Li, X., Z. Jiang, Z. Zhu, Q. Si and Y. Li (2018). Entropy generation analysis for the cavitating head-drop characteristic of a centrifugal pump. Proceedings of the Institution of Mechanical Engineers, Part C: Journal of Mechanical Engineering Science 232(24), 4637-4646.

Liberge, E. and A. Hamdouni (2010). Reduced order modelling method via proper orthogonal decomposition (POD) for flow around an oscillating cylinder. Journal of Fluids and Structures 26 (2), 292-311.

Lumley, J. 1. (1967). The of Inhomogeneous Turbulence. In Atmospheric Turbulence \& Wave Propagation. Yaglom Am, Tatarski VI Nauka. 166-178.

Meyer, K. E., J. M. Pedersen and O. ÖZcan (2007). A turbulent jet in crossflow analysed with proper orthogonal decomposition. Journal of Fluid Mechanics 583, 199-227.

Pavesi, G., G. Cavazzini and G. Ardizzon (2008). Time-frequency characterization of the unsteady phenomena in a centrifugal pump. International Journal of Heat and Fluid Flow 29 (5), 1527-1540.

Prothin, S., H. Djeridi and J. Y. Billard (2014). Coherent and turbulent process analysis of the effects of a longitudinal vortex on boundary layer detachment on a NACA0015 foil. Journal of Fluids and Structures 47, 2-20.

Rudolf, P. and D. Štefan (2012). Decomposition of the swirling flow field downstream of Francis turbine runner. IOP Conference Series: Earth and Environmental Science 15 (6), 062008.

Semlitsch, B. and M. Mihăescu (2016). Flow phenomena leading to surge in a centrifugal compressor. Energy 103, 572-587.

Semlitsch, B., Y. Wang and M. Mihăescu (2014). Flow effects due to pulsation in an internal combustion engine exhaust port. Energy Conversion and Management 86, 520-536.

Sirovich, L. (1987). Turbulence and the dynamics of coherent structures. I. Coherent structures. Quarterly of Applied Mathematics 45, 561571.

Spence, R. and J. Amaral-Teixeira (2008). Investigation into pressure pulsations in a centrifugal pump using numerical methods supported by industrial tests. Computers \& Fluids 37 (6), 690-704.

Stefan, D. and P. Rudolf (2015). Proper Orthogonal Decomposition of Pressure Fields in a Draft Tube Cone of the Francis (Tokke) Turbine 
Z. Y. Liao et al. / JAFM, Vol. 14, No. 1, pp. 98-101, 2021.

Model. Journal of Physics: Conference Series 579, 012002

Tabib, M. V. and J. B. Joshi (2008). Analysis of dominant flow structures and their flow dynamics in chemical process equipment using snapshot proper orthogonal decomposition technique. Chemical Engineering Science 63 (14), 3695-3715

Towne, A., O. T. Schmidt and T. Colonius (2018). Spectral proper orthogonal decomposition and its relationship to dynamic mode decomposition and resolvent analysis. Journal of Fluid Mechanics 847, 821-867.

Wang, H. and H. Tsukamoto (2003). Experimental and Numerical Study of Unsteady Flow in a Diffuser Pump at Off-Design Conditions. Journal of Fluids Engineering 125 (5), 767778.

Willcox, K. (2006). Unsteady flow sensing and estimation via the gappy proper orthogonal decomposition. Computers \& Fluids 35 (2), 208-226.

Witte, M., B. Torner and F.H. Wurm (2018). Analysis of Unsteady Flow Structures in a Radial Turbomachine by Using Proper Orthogonal Decomposition(2018). ASME
Turbo Expo 2018: Turbomachinery Technical Conference and Exposition, Oslo, Norway, June 11-15.

Yang, J., G. Pavesi, G. Cavazzini and S. Q. Yuan (2013). Numerical characterization of pressure instabilities in a vaned centrifugal pump under partload condition. IOP Conference Series: Materials Science and Engineering 52 (2), 022044 .

Yang, J., T. Xie, P. Giorgio, X. Liu and J. Liu (2018). Numerical Study on Rotating Characteristics of Unsteady Flow Inner Pumpturbine in Pump Mode. International Journal of Fluid Machinery and Systems 11 (3), 224233.

Yang, J., T. Xie, X. Liu, Q. Si and J. Liu (2019). Study of Unforced Unsteadiness in Centrifugal Pump at Partial Flow Rates. Journal of Thermal Science, 1-12.

Yuan, S., J. Yang, J. Yuan, Y. Luo and J. Pei (2012). Experimental investigation on the flow-induced noise under variable conditions for centrifugal pumps. Chinese Journal of Mechanical Engineering 25 (3), 456-462. 